\title{
A New Method for the Synthesis of Diversely Functionalized Imidazoles from $\mathrm{N}$-Acylated $\alpha$-Aminonitriles
}

\author{
Yong-Li Zhong*, Jaemoon Lee, Robert A. Reamer, and David Askin \\ Department of Process Research, Merck Research Laboratories, P.O. Box 2000, \\ Rahway, New Jersey 07065

\section{Supporting Information}

Analytical methods: All reactions were carried out under nitrogen. Flash chromatography was carried out with EM science Silica gel 60 (neutral, 230-400 mesh). ${ }^{1} \mathrm{H}$ NMR and ${ }^{13} \mathrm{C}$ NMR spectra were recorded on a Bruker Avance 400 NMR Spectrometer with chemical shifts reported in ppm relative to the residual deuterated solvent. IR spectra were recorded on a Nicolet Margna FT-IR Spectrometer 560. HPLC analysis was performed on a Hewlett Packard 1100 MSD instrument. HRMS was recorded on a Micromass QT of Ultima API US mass spectrometer by ESI.

General experimental procedures for the preparation of $N$-acylated $\alpha$-aminonitrile (Scheme $1 \&$ table 1): To a solution of carboxylic acid acid (0.0200 mol) was added EDC (1.5 equiv), 2aminoacetonitrile hydrochloride (1.1 equiv), a catalytic amount of DMAP, and triethylamine (1.1 equiv). The resulting mixture was stirred at room temperature for $4-16 \mathrm{~h}$. The reaction mixture was quenched with water $(50 \mathrm{~mL})$. After phase separation, the organic layer was washed with $2 \mathrm{~N} \mathrm{HCl} \mathrm{(50}$ $\mathrm{mL})$, water $(30 \mathrm{~mL})$, brine $(30 \mathrm{~mL})$, dried over $\mathrm{MgSO}_{4}$, and concentrated. The solid was recrystallized from $\mathrm{MeOH} /$ water or purified by silica gel chromatography to afford desired $N$-acylated $\alpha$ aminonitriles.

General experimental procedures for the conversion of $\mathrm{N}$-acylated $\alpha$-aminonitrile to 2,4disubstituted-5-haloimidazole (Scheme $1 \&$ 3, and table 1): A solution of $N$-acylated $\alpha$ aminonitrile (5.000 mmoles) and triphenylphosphine $(12.5$ mmoles $)$ in acetonitrile $(50 \mathrm{~mL})$ was treated with carbon tetrachloride $(12.5$ mmoles $)$ at $45{ }^{\circ} \mathrm{C}$ for 16 hours. The reaction mixture was concentrated. The residue was dissolved in methylene chloride $(55 \mathrm{~mL})$. To the solution was added 
$0.5 \mathrm{~N}$ sodium hydroxide $(50 \mathrm{~mL})$. The two-phase mixture was stirred at room temperature for $10 \mathrm{~min}$. After phase separation, the organic layer was washed with water $(2 \times 20 \mathrm{~mL})$, brine $(20 \mathrm{~mL})$, and concentrated. The residue was purified by flash chromatography (silica gel, hexane : EtOAc : TEA = 5 $: 1: 0.05)$ to give desired product.

$N$-[Cyano(phenyl)methyl]benzamide $(4)^{1}, N$-(Cyanomethyl)benzamide $(7 a)^{2}, N$-(Cyanomethyl)-3methoxybenzamide $(10 a)^{3}, N$-(Cyanomethyl)-4-methoxybenzamide $(11 a)^{4},(2 E)-N$-(Cyanomethyl)3-phenylacrylamide (12a) $)^{5}$, and $N$-(Cyanomethyl)pentanamide (18a) ${ }^{6}$ are known compounds.

\section{5-Chloro-2,4-diphenyl-1H-imidazole $(6)^{7}$}

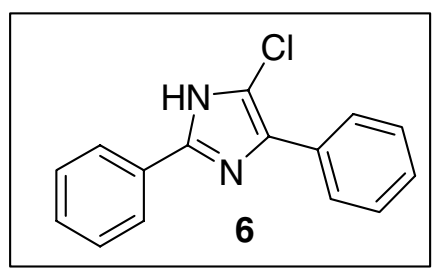

Conditions: $N$-acylated $\alpha$-aminonitrile $4(236.3 \mathrm{mg}, 1.00 \mathrm{mmol}), 45^{\circ} \mathrm{C}, 4 \mathrm{~h}$. Flash chromatography (silica gel, hexane: $\mathrm{CH}_{2} \mathrm{Cl}_{2}:$ EtOAc $\left.=4: 1: 1\right)$ afforded imidazole 6 (193.2 $\left.\mathrm{mg}, 76 \%\right)$ as colorless needles, mp 223.5-225.0 ${ }^{\circ} \mathrm{C}$ (lit. $\left.{ }^{1} 228.5-230{ }^{\circ} \mathrm{C}\right) .{ }^{1} \mathrm{H}$ NMR (400 MHz, DMSO-d 6 ) $\delta 12.88$ (br s, $1 \mathrm{H}$ ), $8.01(\mathrm{dd}, J=8.2,1.0 \mathrm{~Hz}, 2 \mathrm{H}), 7.85$ (bd, $J=7.9 \mathrm{~Hz}, 2 \mathrm{H}), 7.53-7.47$ (m, $4 \mathrm{H})$, 7.42-7.36 (m, $2 \mathrm{H})$.

\section{5-Chloro-2-phenyl-1H-imidazole (7b)}

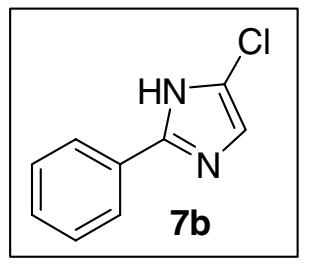

Conditions: $N$-acylated $\alpha$-aminonitrile 7a $(160.2 \mathrm{mg}, 1.00 \mathrm{mmol}), 45^{\circ} \mathrm{C}, 4 \mathrm{~h}$. Flash chromatography (silica gel, hexane: $\mathrm{CH}_{2} \mathrm{Cl}_{2}$ : EtOAc $\left.=4: 1: 1\right)$ afforded imidazole $7 \mathbf{b}(92.5 \mathrm{mg}, 52 \%$ ) as white needles: mp 188.0-188.5 ${ }^{\circ} \mathrm{C}$. IR (thin film) $v_{\max } 3150,2725,1459,1403,1129,982,773,665 \mathrm{~cm}^{-1} .{ }^{1} \mathrm{H}$ NMR $\left(400 \mathrm{MHz}, \mathrm{CDCl}_{3}\right) \delta 9.55$ (br s, $\left.1 \mathrm{H}\right), 7.84-7.77$ (m, $\left.2 \mathrm{H}\right), 7.48-7.35$ (m, $\left.3 \mathrm{H}\right), 7.01$ (s, $\left.1 \mathrm{H}\right) .{ }^{13} \mathrm{C} \mathrm{NMR}$ $\left(100 \mathrm{MHz}, \mathrm{CDCl}_{3}\right) \delta 145.6,129.3(2 \mathrm{C})$ 129.2, 129.0, 125.2 (2 C), 122.1, 112.8. HRMS (ESI) calculated for $\mathrm{C}_{9} \mathrm{H}_{8} \mathrm{ClN}_{2}(\mathrm{M}+\mathrm{H})^{+} 179.0376$, found 179.0374 . 


\section{$N$-(Cyanomethyl)-2-methoxybenzamide (8a)}

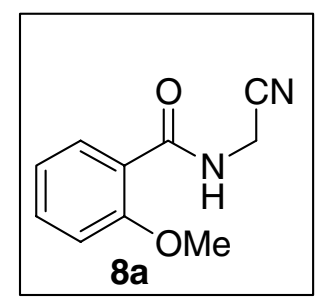

Conditions: $o$-Anisic acid $(4.56 \mathrm{~g}, 30.0 \mathrm{mmol}), \mathrm{rt}, 16 \mathrm{~h}$. Recrystallization from MeOH/water afforded 8a $(5.02 \mathrm{~g}, 88 \%)$ as colorless needles: $\mathrm{mp} 87.0-88.5^{\circ} \mathrm{C}$. IR (thin film) $v_{\max } 3366,3005,2942,2261$, 1642, 1598, 1521, 1480, 1299, 1021, $753 \mathrm{~cm}^{-1} .{ }^{1} \mathrm{H}$ NMR (400 MHz, $\mathrm{CDCl}_{3}$ ) $\delta 8.37$ (br t, $J=5.6 \mathrm{~Hz}, 1$ H), 8.13 (dd, $J=8.0,2.0 \mathrm{~Hz}, 1 \mathrm{H}), 7.44$ (td, $J=8.0,2.0 \mathrm{~Hz}, 1 \mathrm{H}), 7.02$ (t, $J=8.0 \mathrm{~Hz}, 1 \mathrm{H}), 6.94$ (d, $J=$ $8.0 \mathrm{~Hz}, 1 \mathrm{H}), 4.34(\mathrm{~d}, J=5.6 \mathrm{HZ}, 2 \mathrm{H}), 3.93(\mathrm{~s}, 3 \mathrm{H}) .{ }^{13} \mathrm{C} \mathrm{NMR}\left(100 \mathrm{MHz}, \mathrm{CDCl}_{3}\right) \delta$ 165.6, 157.7, $133.8,132.2,121.3,119.6,116.7,111.5,56.1,27.9$. HRMS (ESI) calculated for $\mathrm{C}_{10} \mathrm{H}_{10} \mathrm{~N}_{2} \mathrm{O}_{2}(\mathrm{M}+\mathrm{H})^{+}$ 191.0820, found 191.0816.

\section{5-Chloro-2-(2-methoxyphenyl)-1H-imidazole (8b)}

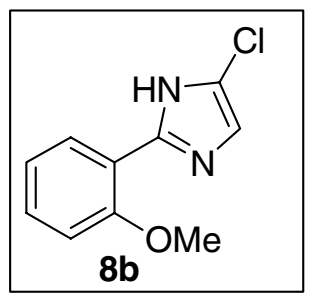

Conditions: $N$-acylated $\alpha$-aminonitrile $8 \mathrm{a}(190.2 \mathrm{mg}, 1.00 \mathrm{mmol}), 45^{\circ} \mathrm{C}, 4.5 \mathrm{~h}$. Flash chromatography (silica gel, hexane: EtOAc $=3: 1)$ afforded imidazole $8 \mathbf{b}(149.0 \mathrm{mg}, 71 \%)$ as colorless plates: $\mathrm{mp}$ $136.8-137.7^{\circ} \mathrm{C}$. IR (thin film) $v_{\max } 3268,1585,1540,1496,1470,1436,1302,1255,1095,1024,963$, 766, 745, $700 \mathrm{~cm}^{-1}$. ${ }^{1} \mathrm{H}$ NMR (400 MHz, $\left.\mathrm{CDCl}_{3}\right) \delta 10.49$ (br s, $\left.1 \mathrm{H}\right), 8.51(\mathrm{dd}, J=7.7,1.8 \mathrm{~Hz}, 1 \mathrm{H})$. $7.34(\mathrm{td}, J=8.8,1.8 \mathrm{~Hz}, 1 \mathrm{H}), 7.08$ ( td, $J=7.7,1.8 \mathrm{~Hz}, 1 \mathrm{H}), 7.01$ (dd, $J=8.8,1.8 \mathrm{~Hz}, 1 \mathrm{H}), 6.97$ (s, 1 $\mathrm{H}), 4.02(\mathrm{~s}, 3 \mathrm{H}) .{ }^{13} \mathrm{C} \mathrm{NMR}\left(100 \mathrm{MHz}, \mathrm{CDCl}_{3}\right) \delta 155.8,143.6,130.0,129.9,128.5,121.7,117.4$, 111.3, 111.2, 55.8. HRMS (ESI) calculated for $\mathrm{C}_{10} \mathrm{H}_{10} \mathrm{ClN}_{2} \mathrm{O}(\mathrm{M}+\mathrm{H})^{+} 209.0482$, found 209.0484.

\section{$N$-(Cyanomethyl)-2-bromobenzamide (9a)}

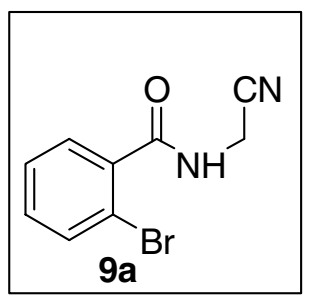

Conditions: 2-Bromobenzoic acid (6.03 g, $30.0 \mathrm{mmol}), \mathrm{rt}, 16 \mathrm{~h}$. Recrystallization from MeOH/water afforded 9a $(6.45 \mathrm{~g}, 90 \%)$ as colorless needles: $\mathrm{mp} 126.0-128.0{ }^{\circ} \mathrm{C}$. IR (thin film) $v_{\max } 3228,3042$, 2934, 2260, 1647, 1588, 1525, 1464, 1315, 1259, 1061, 1027, $751 \mathrm{~cm}^{-1} .{ }^{1} \mathrm{H}$ NMR (400 MHz, $\mathrm{CDCl}_{3}$ ) $\delta: 9.19(\mathrm{t}, J=5.1 \mathrm{~Hz}, 1 \mathrm{H}), 7.68(\mathrm{~d}, J=7.7 \mathrm{~Hz}, 1 \mathrm{H}), 7.48-7.37(\mathrm{~m}, 3 \mathrm{H}), 4.30(\mathrm{~d}, J=5.1 \mathrm{~Hz}, 2 \mathrm{H}) .{ }^{13} \mathrm{C}$ 
$\operatorname{NMR}\left(100 \mathrm{MHz}, \mathrm{CDCl}_{3}\right) \delta: 168.1,137.9,133.4,132.0,129.3,128.2,119.3,117.7,27.8 . \mathrm{MS}$ (FAB) $m / z 239\left(\mathrm{M}^{+}+\mathrm{H}\right), 241\left(\mathrm{M}^{+}+2+\mathrm{H}\right)$. Anal. Calcd for $\mathrm{C}_{9} \mathrm{H}_{7} \mathrm{BrN}_{2} \mathrm{O}: \mathrm{C}, 45.22 ; \mathrm{H}, 2.95$. Found: $\mathrm{C}, 45.25$; H, 3.00.

\section{5-Chloro-2-(2-bromophenyl)-1H-imidazole (9b)}

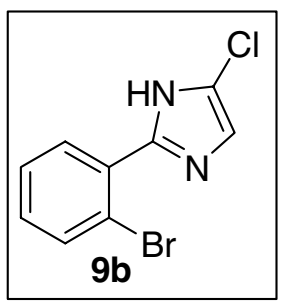

Conditions: $N$-acylated $\alpha$-aminonitrile 9a $(239.1 \mathrm{mg}, 1.00 \mathrm{mmol}), 45^{\circ} \mathrm{C}, 4.5 \mathrm{~h}$. Flash chromatography (silica gel, hexane: EtOAc = 3: 1) afforded imidazole 9b $(189.0 \mathrm{mg}, 74 \%)$ as light yellow needles: $\mathrm{mp}$ 168.1-168.5 ${ }^{\circ} \mathrm{C}$. IR (thin film) $v_{\max } 3056,1653,1559,1506,1463,1436,1092,974,728 \mathrm{~cm}^{-1} .{ }^{1} \mathrm{H}$ NMR $\left(400 \mathrm{MHz} \mathrm{CDCl}_{3}\right) \delta 11.68($ br s, $1 \mathrm{H}), 7.80(\mathrm{dd}, J=7.8,1.7 \mathrm{~Hz}, 1 \mathrm{H}), 7.68(\mathrm{dd}, J=7.8 \mathrm{~Hz}, 1.1 \mathrm{~Hz}, 1$ $\mathrm{H}), 7.45(\mathrm{td}, J=7.8,1.1 \mathrm{~Hz}, 1 \mathrm{H}), 7.32(\mathrm{td}, J=7.8,1.7 \mathrm{~Hz}, 1 \mathrm{H}), 7.25(\mathrm{~s}, 1 \mathrm{H}) .{ }^{13} \mathrm{C} \mathrm{NMR}(100 \mathrm{MHz}$, $\left.\mathrm{CDCl}_{3}\right) \delta 143.4,133.6,131.6,131.3,130.5,129.3,127.7,120.3,113.6$. HRMS (ESI) calculated for $\mathrm{C}_{9} \mathrm{H}_{7} \mathrm{BrClN}_{2}(\mathrm{M}+\mathrm{H})^{+} 256.9481$, found 256.9474 .

\section{5-Chloro-2-(3-mthoxyphenyl)-1H-imidazole (10b)}

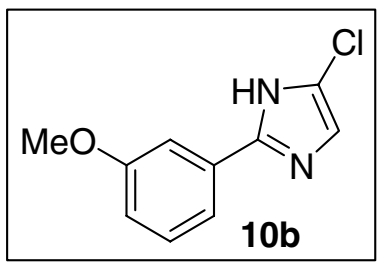

Conditions: $N$-acylated $\alpha$-amino nitrile 10a $(190.2 \mathrm{mg}, 1.00 \mathrm{mmol}), 45{ }^{\circ} \mathrm{C}, 16 \mathrm{~h}$. Flash chromatography (silica gel, hexane: EtOAc $=3: 1)$ afforded imidazole $\mathbf{1 0 b}(83.1 \mathrm{mg}, 40 \%)$ as a white solid: $\mathrm{mp} 169.5-170.0^{\circ} \mathrm{C}$. IR (thin film) $v_{\max } 3053,1653,1558,1506,1471,1464,1437,1381,1285$, 1132, 1036, 859, 755, $692 \mathrm{~cm}^{-1} .{ }^{1} \mathrm{H}$ NMR (400 MHz, $\mathrm{CDCl}_{3}$ ) $\delta 11.23$ (br s, $\left.1 \mathrm{H}\right), 7.36$ (br s, $1 \mathrm{H}$ ), 7.33 (bd, $J=7.8 \mathrm{~Hz}, 1 \mathrm{H}), 7.21(\mathrm{t}, J=7.8 \mathrm{~Hz}, 1 \mathrm{H}), 7.00(\mathrm{~s}, 1 \mathrm{H}), 6.86(\mathrm{dd}, J=7.8,2.4 \mathrm{~Hz}, 1 \mathrm{H}), 3.67$ (s, 3 H). ${ }^{13} \mathrm{C}$ NMR $\left(100 \mathrm{MHz}, \mathrm{CDCl}_{3}\right) \delta 160.0,145.7,130.4,129.9,129.4,117.4,115.8,113.5,110.1,55.1$. HRMS (ESI) calculated for $\mathrm{C}_{10} \mathrm{H}_{10} \mathrm{ClN}_{2} \mathrm{O}(\mathrm{M}+\mathrm{H})^{+} 209.0482$, found 209.0486. 


\section{5-Chloro-2-(4-methoxyphenyl)-1H-imidazole (11b)}

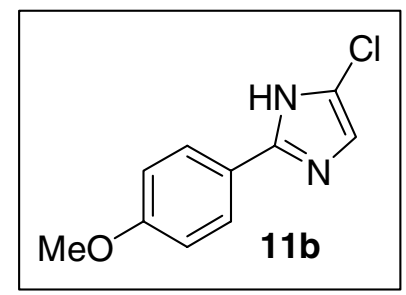

Conditions: $N$-acylated $\alpha$-amino nitrile 11a $(190.2 \mathrm{mg}, 1.00 \mathrm{mmol}), 45^{\circ} \mathrm{C}, 4 \mathrm{~h}$. Flash chromatography (silica gel, hexane: $\mathrm{CH}_{2} \mathrm{Cl}_{2}:$ EtOAc $\left.=3: 1: 1\right)$ afforded imidazole $\mathbf{1 1 b}(151.1 \mathrm{mg}, 72 \%)$ as colorless needles: $m p$ 189.9-190.9 ${ }^{\circ} \mathrm{C}$. IR (thin film) $v_{\max } 3153,2941,2861,1615,1515,1464,1437,1250$, 1127, 843, $735 \mathrm{~cm}^{-1} .{ }^{1} \mathrm{H}$ NMR (400 MHz, $\left.\left(\mathrm{CD}_{3}\right)_{2} \mathrm{CO}\right) \delta 11.77$ (br s, $1 \mathrm{H}$ ), 7.87 (td, $J=8.9,2.9 \mathrm{~Hz}, 2 \mathrm{H}$ ), $7.14(\mathrm{~s}, 1 \mathrm{H}), 6.99(\mathrm{td}, J=8.9,2.8 \mathrm{~Hz}, 2 \mathrm{H}), 3.83(\mathrm{~s}, 3 \mathrm{H}) \cdot{ }^{13} \mathrm{C} \mathrm{NMR}\left(100 \mathrm{MHz},\left(\mathrm{CD}_{3}\right)_{2} \mathrm{CO}\right) \delta 160.2$, 145.3, 129.2, 126.4 (2 C), 122.8, 114.1 (2 C), 112.6, 54.7. HRMS (ESI) calculated for $\mathrm{C}_{10} \mathrm{H}_{10} \mathrm{ClN}_{2} \mathrm{O}(\mathrm{M}$ $+\mathrm{H})^{+}$209.0482, found 209.0476.

\section{5-Chloro-2-[(E)-2-phenylvinyl]-1H-imidazole (12b)}

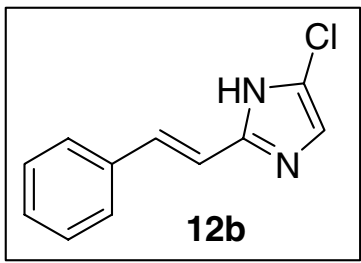

Conditions: $N$-acylated $\alpha$-amino nitrile 12a $(186.2 \mathrm{mg}, 1.00 \mathrm{mmol}), 45{ }^{\circ} \mathrm{C}, 16 \mathrm{~h}$. Flash chromatography (silica gel, hexane: EtOAc $=3: 1)$ afforded imidazole $\mathbf{1 2 b}(144.0 \mathrm{mg}, 70 \%)$ as a light yellow solid: $\mathrm{mp} 181.2-182.9^{\circ} \mathrm{C}$. IR (thin film) $v_{\max } 3051,1653,1558,1506,1464,1420,1121,961$, $748 \mathrm{~cm}^{-1} .{ }^{1} \mathrm{H}$ NMR $\left(400 \mathrm{MHz},\left(\mathrm{CD}_{3}\right)_{2} \mathrm{CO}\right) \delta 11.62(\mathrm{br} \mathrm{s}, 1 \mathrm{H}), 7.53(\mathrm{~d}, J=7.4 \mathrm{~Hz}, 2 \mathrm{H}), 7.4-7.31(\mathrm{~m}, 3$ $\mathrm{H}), 7.27(\mathrm{t}, J=7.2 \mathrm{~Hz}, 1 \mathrm{H}), 7.14(\mathrm{~s}, 1 \mathrm{H}), 6.99(\mathrm{~d}, J=16.4 \mathrm{~Hz}, 1 \mathrm{H}) .{ }^{13} \mathrm{C} \mathrm{NMR}\left(100 \mathrm{MHz},\left(\mathrm{CD}_{3}\right)_{2} \mathrm{CO}\right)$ $\delta$ 145.6, 137.4, 132.0, 130.7, 129.7 (2 C), 129.2, 127.6 (2 C), 117.3, 113.8. HRMS (ESI) calculated for $\mathrm{C}_{11} \mathrm{H}_{10} \mathrm{ClN}_{2}(\mathrm{M}+\mathrm{H})^{+}$205.0533, found 205.0529 .

\section{5-Bromo-2-[(E)-2-phenylvinyl]-1H-imidazole (13b)}

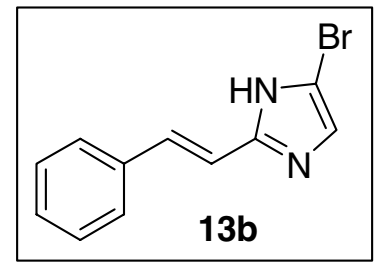

Conditions: $N$-acylated $\alpha$-amino nitrile 13a $(186.2 \mathrm{mg}, 1.00 \mathrm{mmol}), 45{ }^{\circ} \mathrm{C}, 16 \mathrm{~h}$. Flash chromatography (silica gel, hexane: EtOAc $=3: 1)$ afforded imidazole $13 \mathbf{b}(63.0 \mathrm{mg}, 25 \%)$ as a light yellow solid: $\mathrm{mp} 143.0-145.0^{\circ} \mathrm{C}$. IR (thin film) $v_{\max } 3029,1653,1559,1506,1464,1417,1243,1114$, 
963, 907, 841, 751, 736, $688 \mathrm{~cm}^{-1} .{ }^{1} \mathrm{H}$ NMR (400 MHz, $\mathrm{CDCl}_{3}$ ) $\delta 11.63$ (br s, $\left.1 \mathrm{H}\right), 7.58-7.46$ (m, $2 \mathrm{H}$ ), 7.40-7.23 (m, $4 \mathrm{H}), 7.18(\mathrm{~s}, 1 \mathrm{H}), 6.99(\mathrm{~d}, J=16.4 \mathrm{~Hz}, 1 \mathrm{H}) .{ }^{13} \mathrm{C} \mathrm{NMR}\left(100 \mathrm{MHz}, \mathrm{CDCl}_{3}\right) \delta 145.9$, 136.4, 131.0, 130.0, 128.8 (2 C), 128.2, 126.9, 126.6 (2 C), 116.2. HRMS (ESI) calculated for $\mathrm{C}_{11} \mathrm{H}_{10} \mathrm{BrN}_{2}(\mathrm{M}+\mathrm{H})^{+} 249.0027$, found 249.0031.

\section{N-(1-Cyano-3-phenylpropyl)-2-naphthamide (14a)}

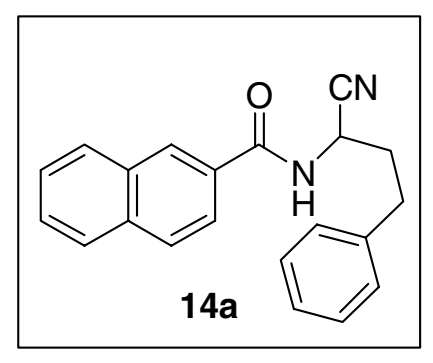

Conditions: 2-Naphthoic acid (3.44 g, $20.0 \mathrm{mmol})$, rt, 16 h. Recrystallization from MeOH/water afforded 14a $(5.01 \mathrm{~g}, 87 \%)$ as white needles: $\mathrm{mp} 157.0-158.5^{\circ} \mathrm{C}$. IR (thin film) $\mathrm{v}_{\max } 3253,2263,1641$, 1523, 1500, 1317, 825, $696 \mathrm{~cm}^{-1} .{ }^{1} \mathrm{H}$ NMR (400 MHz, $\mathrm{CDCl}_{3}$ ) $\delta 8.08$ (br s, $\left.1 \mathrm{H}\right)$, 7.86-7.80 (m, $3 \mathrm{H}$ ), $7.67(\mathrm{dd}, J=8.7,1.8 \mathrm{~Hz}, 1 \mathrm{H}), 7.61-7.52(\mathrm{~m}, 2 \mathrm{H}), 7.36-7.25(\mathrm{~m}, 5 \mathrm{H}), 6.71(\mathrm{~d}, J=8.4 \mathrm{~Hz}, 1 \mathrm{H}), 5.19$ $(\mathrm{q}, J=6.9 \mathrm{~Hz}, 1 \mathrm{H}), 3.01-2.82(\mathrm{~m}, 2 \mathrm{H}), 2.31(\mathrm{q}, J=7.6 \mathrm{~Hz}, 2 \mathrm{H}) .{ }^{13} \mathrm{C} \mathrm{NMR}\left(100 \mathrm{MHz}, \mathrm{CDCl}_{3}\right)$ $\delta 166.7,139.5,135.0,132.4,129.1,129.0$ (2 C), 128.9, 128.6, 128.5 (2 C), 128.1, 127.9, 127.8, 127.0, 126.8, 123.3, 118.5, 40.9, 34.4, 31.8. HRMS (ESI) calculated for $\mathrm{C}_{21} \mathrm{H}_{18} \mathrm{~N}_{2} \mathrm{O}(\mathrm{M}+\mathrm{H})^{+} 315.1497$, found 315.1498 .

\section{5-Chloro-2-(2-naphthyl)-4-(2-phenylethyl)-1H-imidazole (14b)}

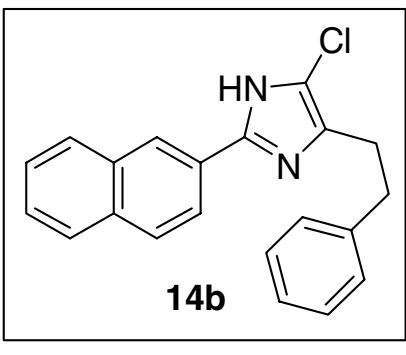

Conditions: $N$-acylated $\alpha$-aminonitrile $14 \mathbf{a}(314.2 \mathrm{mg}, 1.00 \mathrm{mmol}), 45^{\circ} \mathrm{C}, 12 \mathrm{~h}$. Flash chromatography ( silica gel, hexane: EtOAc = 5: 1) afforded imidazole 14b $(222.0 \mathrm{mg}, 67 \%)$ as colorless needles: $\mathrm{mp}$ 193.0-194.9 ${ }^{\circ} \mathrm{C}$. IR (thin film) $v_{\max } 3057,3023,1653,1559,1506,1462,1406,1102,989,902,862$, 824, 754, $702 \mathrm{~cm}^{-1} .{ }^{1} \mathrm{H}$ NMR (400 MHz, $\mathrm{CDCl}_{3}$ ) $\delta 9.71$ (br s, $\left.1 \mathrm{H}\right), 8.05$ (s, $\left.1 \mathrm{H}\right)$, 7.80-7.66 (m, $\left.4 \mathrm{H}\right)$, 7.49-7.43 (m, 2 H), 7.38-7.26 (m, 3 H), 7.20 (d, J = 7.3 Hz, 2 H), 3.05-2.95 (m, 4 H). ${ }^{13} \mathrm{C}$ NMR (100 $\left.\mathrm{MHz}, \mathrm{CDCl}_{3}\right) \delta 157.8,143.8,140.7,133.3,133.2,128.8,128.7,128.6,128.2,127.7,127.5,126.7$, 
126.6, 126.5, 125.9, 123.7, 122.4, 35.0, 25.8. HRMS (ESI) calculated for $\mathrm{C}_{21} \mathrm{H}_{18} \mathrm{ClN}_{2}(\mathrm{M}+\mathrm{H})^{+}$ 333.1158 , found 333.1147 .

\section{$\mathrm{N}$-(Cyanometryl)abieta-7,13-dien-18-amide (15a)}

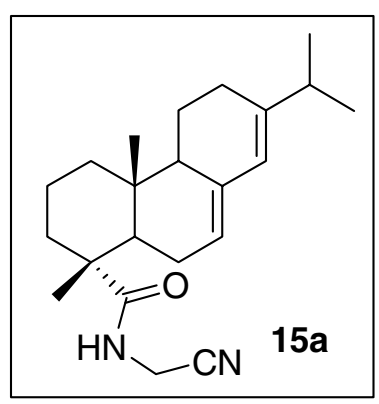

Conditions: Abietic acid (9.07 g, $30.0 \mathrm{mmol})$, rt, 16 h. Flash chromatography (silica gel, hexane: EtOAc $=4: 1)$ afforded $N$-acylated $\alpha$-aminonitrile $\mathbf{1 5 a}(5.10 \mathrm{~g}, 50 \%)$ as white solid: $\mathrm{mp} 92.5-95.5{ }^{\circ} \mathrm{C}$. IR (thin film) $v_{\max } 3360,2917,2865,1633,1507,1472,1429,1273,1197,1002,885 \mathrm{~cm}^{-1} .{ }^{1} \mathrm{H}$ NMR $\left(400 \mathrm{MHz}, \mathrm{CDCl}_{3}\right) \delta 6.39(\mathrm{br} \mathrm{t}, J=6.1 \mathrm{~Hz}, 1 \mathrm{H}), 5.76(\mathrm{~s}, 1 \mathrm{H}), 5.37-5.33(\mathrm{~m}, 1 \mathrm{H}), 4.14(\mathrm{t}, J=6.1 \mathrm{~Hz}$, $2 \mathrm{H}), 2.25-2.19(\mathrm{~m}, 1 \mathrm{H}), 2.15-1.56(\mathrm{~m}, 11 \mathrm{H}), 1.29(\mathrm{~s}, 3 \mathrm{H}), 1.27-1.12(\mathrm{~m}, 3 \mathrm{H}), 1.01(\mathrm{~d}, J=6.8 \mathrm{~Hz}, 3$ $\mathrm{H}), 1.00(\mathrm{~d}, J=6.8 \mathrm{~Hz}, 3 \mathrm{H}), 0.84(\mathrm{~s}, 3 \mathrm{H}) .{ }^{13} \mathrm{C} \mathrm{NMR}\left(100 \mathrm{MHz}, \mathrm{CDCl}_{3}\right) \delta 178.8,145.4,135.6,122.3$, 120.0, 116.4, 51.0, 46.6, 45.8, 38.2, 37.5, 34.9, 34.7, 27.9, 27.4, 25.1, 22.4, 21.4, 20.8, 18.1, 16.8, 14.1. HRMS (ESI) calculated for $\mathrm{C}_{22} \mathrm{H}_{32} \mathrm{~N}_{2} \mathrm{O}(\mathrm{M}+\mathrm{H})^{+} 341.2593$, found 341.2592.

5-Chloro-2-[(1,4a-cis)-7-isopropyl-1,4a-dimethyl-1,2,3,4,4a,4b,5,6,10,-

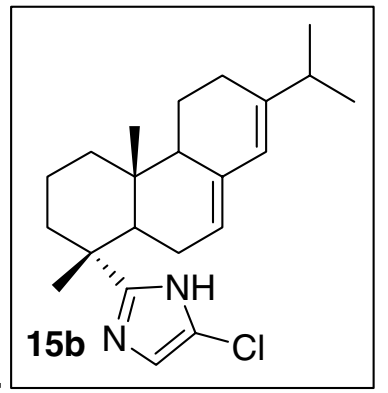

\section{0a-decahydrophenanthren-1-yl]-1H-imidazole (15b)}

Conditions: $N$-acylated $\alpha$-aminonitrile $15 \mathbf{a}(340.5 \mathrm{mg}, 1.00 \mathrm{mmol}), 45^{\circ} \mathrm{C}, 13 \mathrm{~h}$. Flash chromatography (silica gel, hexane: $\mathrm{CH}_{2} \mathrm{Cl}_{2}:$ EtOAc = 3: 1: 1) afforded imidazole 15b (135.9 mg, 38\%) as white needles: $\mathrm{mp} 201.5-202.9^{\circ} \mathrm{C}$. IR (thin film) $v_{\max } 3159,1653,1541,1464,1411,1255,1208,1095,827$, $729 \mathrm{~cm}^{-1} .{ }^{1} \mathrm{H}$ NMR $\left(400 \mathrm{MHz}, \mathrm{CDCl}_{3}\right) \delta 8.79$ (br s, $\left.1 \mathrm{H}\right), 6.77(\mathrm{~s}, 1 \mathrm{H}), 5.74(\mathrm{~s}, 1 \mathrm{H}), 5.29(\mathrm{~d}, J=4.8$ $\mathrm{Hz}, 1 \mathrm{H}), 2.26-2.17(\mathrm{~m}, 1 \mathrm{H}), 2.16-1.49$ (m, $11 \mathrm{H}), 1.46(\mathrm{~s}, 3 \mathrm{H}), 1.35-1.15(\mathrm{~m}, 3 \mathrm{H}), 1.01(\mathrm{~d}, J=6.8$ $\mathrm{Hz}, 3 \mathrm{H}), 1.00(\mathrm{~d}, J=6.8 \mathrm{~Hz}, 3 \mathrm{H}), 0.89$ (s, $3 \mathrm{H}) .{ }^{13} \mathrm{C} \mathrm{NMR}\left(100 \mathrm{MHz}, \mathrm{CDCl}_{3}\right) \delta$ 155.7, 145.4, 135.6, 
$124.5,122.2,120.3,110.3,50.9,48.8,40.2,40.1,38.3,35.2,34.8,27.4,25.0,22.5,21.4,20.8,18.4$, 17.7, 14.2. HRMS (ESI) calculated for $\mathrm{C}_{22} \mathrm{H}_{32} \mathrm{ClN}_{2}(\mathrm{M}+\mathrm{H})^{+} 359.2254$, found 359.2258.

\section{2-[(1,3-cis)-3-Acetyl-2,2-dimethylcyclobutyl]- $N$ -}

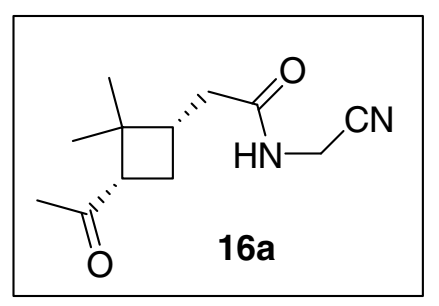
(cyanometryl)acetamide (16a)

Conditions: cis-Pinonic acid (3.68 g, $20.0 \mathrm{mmol})$, rt, $4 \mathrm{~h}$. Recrystallization from MeOH/water afforded 16a (3.82 g, 86\%) as white needles: $\mathrm{mp}$ 73.0-74.0 ${ }^{\circ} \mathrm{C}$. IR (thin film) $\mathrm{v}_{\max } 3255,3052,2989$, 2953, 2865, 2261, 1703, 1651, 1531, 1462, 1375, 1359, 1316, 1270, 1159, 1034, $698 \mathrm{~cm}^{-1} .{ }^{1} \mathrm{H}$ NMR $\left(400 \mathrm{MHz}, \mathrm{CDCl}_{3}\right) \delta 6.85$ (br s, $\left.1 \mathrm{H}\right), 4.18(\mathrm{dd}, J=17.5,5.8 \mathrm{HZ}, 1 \mathrm{H}), 4.13(\mathrm{dd}, J=17.5,5.8 \mathrm{HZ}, 1$ H), $2.93(\mathrm{dd}, J=9.9,7.6 \mathrm{~Hz}, 1 \mathrm{H}), 2.47-2.39$ (m, $1 \mathrm{H}), 2.28$ (dd, $J=14.5,6.7 \mathrm{~Hz}, 1 \mathrm{H}), 2.18$ (dd, $J=$ 14.5, 8.6 Hz, $1 \mathrm{H}), 2.06$ (s, $3 \mathrm{H}), 2.03-1.88$ (m, $2 \mathrm{H}), 1.34$ (s, $3 \mathrm{H}), 0.85$ (s, $3 \mathrm{H}) .{ }^{13} \mathrm{C} \mathrm{NMR}(100 \mathrm{MHz}$, $\left.\mathrm{CDCl}_{3}\right) \delta 208.3,172.2,116.2,54.1,43.4,38.2,36.7,30.3,30.1,27.3,22.7,17.5$. MS (FAB) $m / z 223$ $\left(\mathrm{M}^{+}+\mathrm{H}\right)$. Anal. Calcd for $\mathrm{C}_{12} \mathrm{H}_{18} \mathrm{~N}_{2} \mathrm{O}_{2}: \mathrm{C}, 64.84 ; \mathrm{H}, 8.16$. Found: $\mathrm{C}, 64.88 ; \mathrm{H}, 8.42$.

\section{1-\{(1,3)-cis-3-[(5-Chloro-1H-imidazol-2-yl)methyl]-}

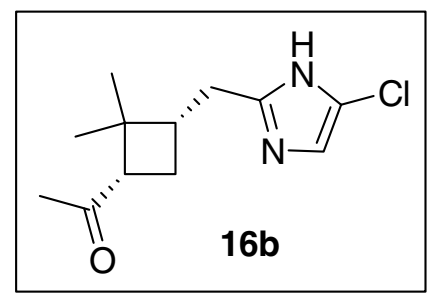

\section{2,2-dimethylcyclobutyl\}ethanone (16b)}

Conditions: $N$-acylated $\alpha$-aminonitrile 16a $(222.3 \mathrm{mg}, 1.00 \mathrm{mmol}), 45^{\circ} \mathrm{C}, 16 \mathrm{~h}$. Flash chromatography (silica gel, toluene: $\left.\mathrm{CH}_{3} \mathrm{CN}=10: 1\right)$ afforded imidazole $16 \mathbf{b}(163.0 \mathrm{mg}, 68 \%$ ) as a white solid: $\mathrm{mp}$ 116.0-117.9 ${ }^{\circ} \mathrm{C}$. IR (thin film) $v_{\max } 3149,3055,1702,1559,1440,1369,1301,1202,1182,1120$, 1100, 966, $723 \mathrm{~cm}^{-1} .{ }^{1} \mathrm{H}$ NMR (400 $\left.\mathrm{MHz}, \mathrm{CDCl}_{3}\right) \delta 10.35$ (br s, $\left.1 \mathrm{~h}\right), 6.79$ (s, $\left.1 \mathrm{H}\right), 2.88(\mathrm{dd}, J=10.0$, $7.5 \mathrm{~Hz}, 1 \mathrm{H}), 2.73$ (dd, $J=14.6,6.3 \mathrm{~Hz}, 1 \mathrm{H}), 2.55$ (dd, $J=14.6,9.3 \mathrm{~Hz}, 1 \mathrm{H}), 2.47-2.36(\mathrm{~m}, 1 \mathrm{H})$, 2.05 (s, $3 \mathrm{H}), 2.02(\mathrm{dd}, J=12.3,10.0 \mathrm{~Hz}, 1 \mathrm{H}), 1.86(\mathrm{dt}, J=12.3,7.5 \mathrm{~Hz}, 1 \mathrm{H}), 1.27$ (s, $3 \mathrm{H}), 0.90$ (s, 3 H). ${ }^{13} \mathrm{C}$ NMR $\left(100 \mathrm{MHz} \mathrm{CDCl}_{3}\right) \delta 208.3,146.2,128.7,111.0,54.1,43.4,40.6,30.3,30.1,29.4,23.1$, 17.4. HRMS (ESI) calculated for $\mathrm{C}_{12} \mathrm{H}_{18} \mathrm{ClN}_{2} \mathrm{O}(\mathrm{M}+\mathrm{H})^{+} 241.1106$, found 241.1109. 


\section{2-[(1,3-cis)-3-Acetyl-2,2-dimethylcyclobutyl]- $N$ -}

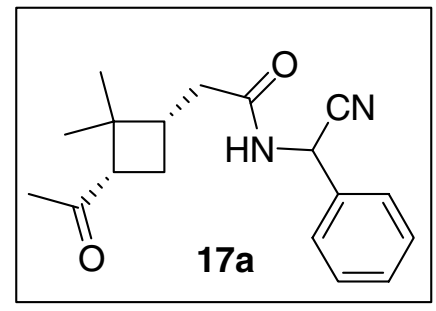

\section{[cyano(phenyl)metryl]acetamide (17a)}

Conditions: cis-Pinonic acid (1.00 g, $5.43 \mathrm{mmol}$ ), rt, $4 \mathrm{~h}$. Flash chromatography (silica gel, hexane: $\left.\mathrm{CH}_{2} \mathrm{Cl}_{2}: \mathrm{EtOAc}=4: 1: 1\right)$ afforded 17a $(1.58 \mathrm{~g}, 98 \%)$ as colorless oil. IR (thin film) $v_{\max } 3245,3033$, $2952,2262,1700,1641,1518,1495,1369,1225,1182,759,696 \mathrm{~cm}^{-1} .{ }^{1} \mathrm{H}$ NMR $\left(400 \mathrm{MHz}, \mathrm{CDCl}_{3}\right) \delta$ 7.46-7.38 (m, $5 \mathrm{H}), 6.88$ (br d, $J=8.4 \mathrm{~Hz}, 0.5 \mathrm{H}), 6.84(\mathrm{br} \mathrm{d}, J=8.4 \mathrm{~Hz}, 0.5 \mathrm{H}), 6.11(\mathrm{~d}, J=8.4 \mathrm{~Hz}$, $0.5 \mathrm{H}), 6.09$ (d, $J=8.4 \mathrm{~Hz}, 0.5 \mathrm{H}), 2.93-2.87$ (m, $1 \mathrm{H}), 2.48-2.39$ (m, $1 \mathrm{H}), 2.33-2.14(\mathrm{~m}, 2 \mathrm{H}), 2.06-$ $1.81(\mathrm{~m}, 5 \mathrm{H}), 1.33(\mathrm{~s}, 1.5 \mathrm{H}), 1.32(\mathrm{~s}, 1.5 \mathrm{H}), 0.84(\mathrm{~s}, 1.5 \mathrm{H}), 0.81(\mathrm{~s}, 1.5 \mathrm{H}) .{ }^{13} \mathrm{C}$ NMR $(100 \mathrm{MHz}$, $\left.\mathrm{CDCl}_{3}\right) \delta 208.1,171.3,133.3,129.4,129.3$ (2 C), 127.0, 126.9, 117.5, 54.1, 43.9, 43.4, 38.4, 36.9, 30.3, 30.1, 22.8, 17.5. HRMS (ESI) calculated for $\mathrm{C}_{12} \mathrm{H}_{22} \mathrm{~N}_{2} \mathrm{O}_{2}(\mathrm{M}+\mathrm{H})^{+}$299.1760, found 299.1764. Anal. Calcd for $\mathrm{C}_{18} \mathrm{H}_{22} \mathrm{~N}_{2} \mathrm{O}_{2}$ : C, 72.46; H, 7.43. Found: C, 72.44; H, 7.33.

\section{1-\{(1,3)-cis-3-[(5-Chloro-4-phenyl-1H-imidazol-2-yl)}

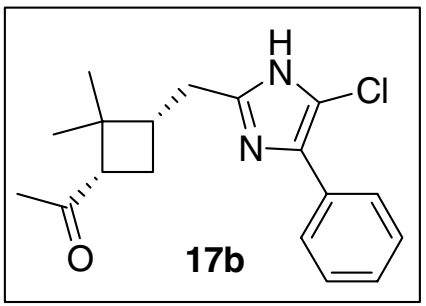

\section{methyl]-2,2-dimethylcyclobutyl\}ethanone (17b)}

Conditions: $N$-acylated $\alpha$-aminonitrile $17 \mathbf{a}(298.4 \mathrm{mg}, 1.00 \mathrm{mmol}), 45^{\circ} \mathrm{C}, 16 \mathrm{~h}$. Flash chromatography (silica gel, toluene: $\left.\mathrm{CH}_{3} \mathrm{CN}=8: 1\right)$ afforded imidazole $\mathbf{1 7 b}(229.0 \mathrm{mg}, 72 \%)$ as a light yellow solid: mp 94.6-95.8 ${ }^{\circ} \mathrm{C}$. IR (thin film) $v_{\text {max }} 3050,1703,1585,1516,1452,1369,1226,1180,983,763,722$, $694 \mathrm{~cm}^{-1} .{ }^{1} \mathrm{H}$ NMR $\left(400 \mathrm{MHz}, \mathrm{CDCl}_{3}\right) \delta 10.91$ (br s, $\left.1 \mathrm{H}\right), 7.68$ (br d, $\left.J=7.6 \mathrm{~Hz}, 2 \mathrm{H}\right), 7.39$ (br t, $J=$ $7.6 \mathrm{HZ}, 2 \mathrm{H}), 7.26$ (br t, $J=7.6 \mathrm{~Hz}, 1 \mathrm{H}), 2.84$ (br t, $J=8.3 \mathrm{~Hz}, 1 \mathrm{H}), 2.72$ (dd, $J=14.4,5.7 \mathrm{~Hz}, 1 \mathrm{H}$ ), $2.54(\mathrm{dd}, J=14.4,9.7 \mathrm{~Hz}, 1 \mathrm{H}), 2.46-2.36(\mathrm{~m}, 1 \mathrm{H}), 2.04$ (d, $J=11.1 \mathrm{~Hz}, 1 \mathrm{H}), 2.01$ (s, $3 \mathrm{H}), 1.80-1.55$ (m, $1 \mathrm{H}), 1.24$ (s, $3 \mathrm{H}), 0.87$ (s, $3 \mathrm{H}) .{ }^{13} \mathrm{C}$ NMR $\left(100 \mathrm{MHz}, \mathrm{CDCl}_{3}\right)$ 8: 208.9, 145.9, 129.0, 128.7 (2 C), 127.3, 125.8 (2 C), 125.1, 124.1, 54.1, 43.5, 40.6, 30.3, 30.0, 29.2, 23.0, 17.4. HRMS (ESI) calculated for $\mathrm{C}_{18} \mathrm{H}_{22} \mathrm{ClN}_{2} \mathrm{O}(\mathrm{M}+\mathrm{H})^{+} 317.1421$, found 317.1426. 


\section{2-Butyl-5-chloro-1H-imidazole (18b)}

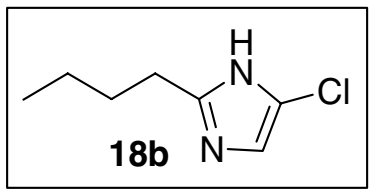

Conditions: $N$-acylated $\alpha$-aminonitrile $18 a(140.0 \mathrm{mg}, 1.00 \mathrm{mmol}), 45^{\circ} \mathrm{C}, 16 \mathrm{~h}$. Flash chromatography (silica gel, hexane: EtOAc = 5: 1) afforded imidazole $18 \mathrm{~b}(108.0 \mathrm{mg}, 68 \%)$ as a white solid: $\mathrm{mp} 61.0$ 63.0 (lit. ${ }^{2} 62-64{ }^{\circ} \mathrm{C}$ ). ${ }^{1} \mathrm{H} \mathrm{NMR}\left(400 \mathrm{MHz}, \mathrm{CDCl}_{3}\right.$ ) $\delta 11.04$ (br s, $\left.1 \mathrm{H}\right), 6.83(\mathrm{~s}, 1 \mathrm{H}), 2.70$ (t, $J=7.9 \mathrm{~Hz}$, 2 H), 1.75-1.65 (m, 2 H), 1.40-1.29 (m, 2 H), 0.90 (t, $J=7.4$ Hz, 3 H).

\section{$N$-[Cyano(phenyl)methyl]-2-(2,5-difluorophenyl)acetamide (19a)}

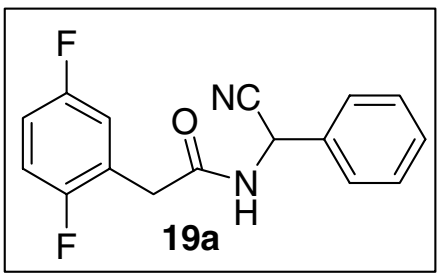

Conditions: 2,5-Difluorobenzylacetic acid (3.44 g, $20.0 \mathrm{mmol})$, rt, $4 \mathrm{~h}$. Recrystallization from $\mathrm{MeOH} /$ water afforded 19a $(3.82 \mathrm{~g}, 86 \%)$ as white needles: $\mathrm{mp} 171.0-172.5^{\circ} \mathrm{C}$. IR (thin film) $v_{\max } 3270$, $3080,3014,2260,2653,1521,1495,1452,1351,1221,1171,1141,1073,879,824,754 \mathrm{~cm}^{-1} .{ }^{1} \mathrm{H}$ NMR (400 MHz, $\mathrm{CDCl}_{3}$ ) $\delta$ 7.45-7.37 (m, $\left.5 \mathrm{H}\right), 7.27-6.95$ (m, $\left.3 \mathrm{H}\right), 6.27(\mathrm{br} \mathrm{d}, J=8.1 \mathrm{~Hz}, 1 \mathrm{H}), 6.12$ $(\mathrm{d}, J=8.1 \mathrm{~Hz}, 1 \mathrm{H}), 3.61(\mathrm{~s}, 2 \mathrm{H}) .{ }^{13} \mathrm{C}$ NMR $\left(100 \mathrm{MHz}, \mathrm{CDCl}_{3}\right) \delta 168.4,158.5(\mathrm{~d}, J=241 \mathrm{~Hz}), 157.2$ (d, $J=240 \mathrm{HZ}), 134.3,129.0$ (2C), 128.9, 127.1 (2 C), 124.4 (dd, $J=19,8 \mathrm{~Hz}), 118.1$ (dd, $J=24,5$ $\mathrm{Hz}), 117.8,116.2(\mathrm{dd}, J=25,9 \mathrm{~Hz}), 115.0(\mathrm{dd}, J=24,9 \mathrm{~Hz}), 44.0,34.9$. MS (FAB) $\mathrm{m} / z 287\left(\mathrm{M}^{+}+\mathrm{H}\right)$. Anal. Calcd for $\mathrm{C}_{16} \mathrm{H}_{12} \mathrm{~F}_{2} \mathrm{~N}_{2} \mathrm{O}$ : C, 67.13; $\mathrm{H}, 4.23$. Found: C, 66.94; H, 4.11.

\section{5-Chloro-2-(2,5-difluorobenzyl)-4-phenyl-1H-imidazole (19b)}

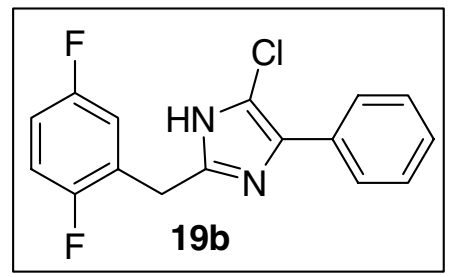

Conditions: $N$-acylated $\alpha$-aminonitrile 19a $(1.430 \mathrm{~g}, 5.00 \mathrm{mmol}), 45{ }^{\circ} \mathrm{C}, 16 \mathrm{~h}$. The residue was purified by flash chromatography (silica gel, hexane: EtOAc $=6: 1)$ to give imidazole $19 \mathrm{~b}(1.125 \mathrm{~g}$, $74 \%$ ) as light yellow needles: $\mathrm{mp} 193.1-193.7^{\circ} \mathrm{C}$. IR (thin film) $v_{\text {max }} 1653,1559,1497,1471,1457$, $1232,1211,1137,981,870,823,762,729,717,689 \mathrm{~cm}^{-1} .{ }^{1} \mathrm{H}$ NMR $\left(400 \mathrm{MHz}, \mathrm{CDCl}_{3}\right) \delta 9.98(\mathrm{br} \mathrm{s}, 1$ H), 7.61 (br d, $J=7.5 \mathrm{~Hz}, 2 \mathrm{H}$ ), 7.41 (br t, $J=7.5 \mathrm{~Hz}, 2 \mathrm{H}$ ), 7.31 (br t, $J=7.5 \mathrm{~Hz}, 1 \mathrm{H}$ ), 7.06-6.86 (m, $3 \mathrm{H}), 4.01(\mathrm{~s}, 2 \mathrm{H}) .{ }^{13} \mathrm{C} \mathrm{NMR}\left(100 \mathrm{MHz}, \mathrm{CDCl}_{3}\right) \delta 158.6(\mathrm{dd}, J=240,2 \mathrm{~Hz}), 156.8(\mathrm{dd}, J=240,2 \mathrm{~Hz})$, 143.5, 139.0, 128.9, 128.4, 127.7, 126.8, 125.8, 125.0 (dd, $J=20,7 \mathrm{~Hz}), 117.5$ (dd, $J=20,4 \mathrm{~Hz})$, 
$116.6(\mathrm{dd}, J=20,10 \mathrm{~Hz}), 115.6(\mathrm{dd}, J=20,10 \mathrm{~Hz}), 28.5$. HRMS (ESI) calculated for $\mathrm{C}_{16} \mathrm{H}_{12} \mathrm{ClF}_{2} \mathrm{~N}_{2}(\mathrm{M}$ $+\mathrm{H})^{+} 305.0657$, found 305.0667 .

\section{$\mathrm{N}$-[2-Benzyloxy)-1-cyanoethyl]pentanamide (21)}

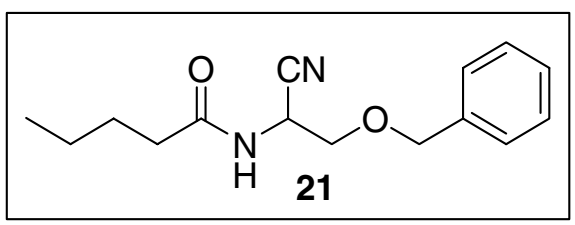

To a solution of $25 \%$ ammonium hydroxide $(16 \mathrm{~mL})$ was added sodium cyanide $(2.04 \mathrm{~g}, 0.0417 \mathrm{~mol})$, and ammonium chloride $(2.63 \mathrm{~g}, 0.0492 \mathrm{~mol})$ with vigorous stirring. Then, the benzyloxyacetaladehyde $(5.00 \mathrm{~g}, 0.0333 \mathrm{~mol})$ was added dropwise within $0.5 \mathrm{~h}$ at room temperature. The mixture was stirred at ambient temperature for $56 \mathrm{~h}$, and then extracted with dichloromethane (40 $\mathrm{mL})$. The organic layer was washed with brine $(20 \mathrm{~mL})$, dried over $\mathrm{MgSO}_{4}$, and filtered. To the filtration was added valeric acid $(3.40 \mathrm{~g}, 0.0333 \mathrm{~mol})$, EDC $(9.58 \mathrm{~g}, 0.0500 \mathrm{~mol})$, and a catalytic amount of DMAP (1.02 g, $0.00833 \mathrm{~mol})$. The resulting mixture was stirred at room temperature for 16 h. The reaction mixture was quenched with water $(50 \mathrm{~mL})$. After phase separation, the organic layer was washed with $2 \mathrm{~N} \mathrm{HCl}(50 \mathrm{~mL})$, water $(30 \mathrm{~mL})$, brine $(30 \mathrm{~mL})$, dried over $\mathrm{MgSO}_{4}$, and concentrated. The residue was purified by flash chromatography (silica gel, hexane: EtOAc $=5: 1$ ) to afford $\beta$-Benzyloxy- $\alpha$-( $N$-butyryl)-aminopropionitrile 21 (6.08 g, 70\% overall) as a white solid: $\mathrm{mp}$ 47.7-48.6 ${ }^{\circ} \mathrm{C}$. IR (thin film) $v_{\max } 3288,2926,1653,1533,1456,1364,1204,1108,1050,742,697 \mathrm{~cm}^{-1}$.

${ }^{1} \mathrm{H}$ NMR $\left(400 \mathrm{MHz}, \mathrm{CDCl}_{3}\right) \delta$ 7.49-7.25 (m, $\left.5 \mathrm{H}\right), 6.28$ (br s, $\left.1 \mathrm{H}\right)$, 5.23-5.03 (m, $\left.1 \mathrm{H}\right), 4.65(\mathrm{~d}, J=$ $12.0 \mathrm{~Hz}, 1 \mathrm{H}), 4.61(\mathrm{~d}, J=12.0 \mathrm{~Hz}, 1 \mathrm{H}), 3.75(\mathrm{dd}, J=9.9,3.3 \mathrm{~Hz}, 1 \mathrm{H}), 3.62$ (dd, $J=9.9,3.8 \mathrm{~Hz}, 1$ $\mathrm{H}), 2.21(\mathrm{t}, J=7.5 \mathrm{~Hz}, 2 \mathrm{H}), 1.70-1.55(\mathrm{~m}, 2 \mathrm{H}), 1.42-1.25(\mathrm{~m}, 2 \mathrm{H}), 0.89(\mathrm{t}, J=7.4 \mathrm{~Hz}, 3 \mathrm{H}) .{ }^{13} \mathrm{C}$ NMR (100 MHz, $\left.\mathrm{CDCl}_{3}\right) \delta 172.5,136.7,128.7$ (2 C), 128.3, 127.9 (2 C), 117.4, 73.7, 68.9, 40.5, 35.8, 27.3, 22.2, 13.7. HRMS (ESI) calculated for $\mathrm{C}_{15} \mathrm{H}_{21} \mathrm{~N}_{2} \mathrm{O}_{2}(\mathrm{M}+\mathrm{H})^{+} 261.1603$, found 261.1611.

\section{5-Benzyloxymethyl-2-butyl-4-chloro-1H-imidazole (22)}

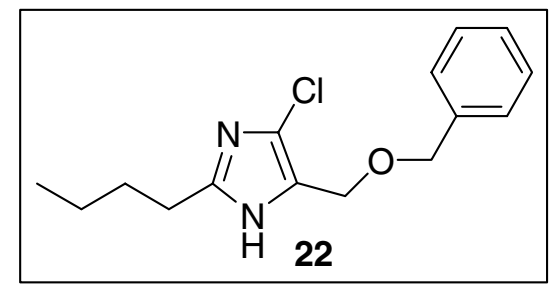

Conditions: $N$-acylated $\alpha$-amino nitrile 21 (781.0 mg, $3.00 \mathrm{mmol}), 45{ }^{\circ} \mathrm{C}, 4 \mathrm{~h}$. The residue was purified by flash chromatography (silica gel, hexane: EtOAc = 5: 1) to give imidazole $22(675.0 \mathrm{mg}$, $81 \%$ ) as a light yellow solid: $\mathrm{mp} 71.7-72.0^{\circ} \mathrm{C}$. IR (thin film) $\mathrm{v}_{\max } 3029,1595,1519,1466,1432,1269$, 1228, 1061, 1037, 913, 892, 853, 806, 735, $695 \mathrm{~cm}^{-1} .{ }^{1} \mathrm{H}$ NMR (400 MHz, $\left.\mathrm{CDCl}_{3}\right) \delta 11.45$ (br s, $\left.1 \mathrm{H}\right)$, 7.32-7.15 (m, $5 \mathrm{H}), 4.51(\mathrm{~s}, 4 \mathrm{H}), 2.59(\mathrm{t}, J=7.6 \mathrm{~Hz}, 2 \mathrm{H}), 1.66-1.55(\mathrm{~m}, 2 \mathrm{H}), 1.35-1.23(\mathrm{~m}, 2 \mathrm{H})$, 
$0.86(\mathrm{t}, J=7.3 \mathrm{~Hz}, 3 \mathrm{H}) .{ }^{13} \mathrm{C} \mathrm{NMR}\left(100 \mathrm{MHz} \mathrm{CDCl}_{3}\right) \delta 148.4,137.5,128.4(2 \mathrm{C}), 128.0,127.9(2 \mathrm{C})$, 127.2, 120.9, 71.9, 60.9, 30.3, 28.3, 22.2, 13.7. HRMS (ESI) calculated for $\mathrm{C}_{15} \mathrm{H}_{20} \mathrm{ClN}_{2} \mathrm{O}(\mathrm{M}+\mathrm{H})^{+}$ 279.1264, found 279.1270.

\section{2-Butyl-4-chloro-5-hydroxymethyl-1H-imidazole (2) ${ }^{9}$}

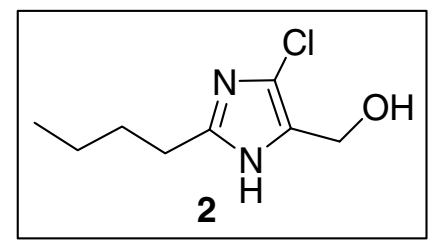

A mixture of 2-butyl-4-chloro-5-benzyloxymethyl-1H-imidazole 22 (139.4 mg, $0.500 \mathrm{mmol})$ and methanesulfonic acid $(1.25 \mathrm{~mL})$ in chloroform $(2.8 \mathrm{~mL})$ was stirred at room temperature for $1 \mathrm{~h}$. The reaction mixture was poured onto ice $(5 \mathrm{~g})$. The resulting solution was neutralized by $5 \mathrm{~N}$ sodium hydroxide to adjust the $\mathrm{pH}$ to 10 . The solution was extracted with MTBE $(2 \times 5 \mathrm{~mL})$. The aqueous layer was extracted with $n$-butanol $(3 \times 5 \mathrm{~mL})$. The combined extractions from $n$-butanol were concentrated and solvent-switched to toluene. The crystalline solid was filtered off, washed with cold water, hexane/MTBE (4: 1), and dried under vacuum to give imidazole $2(84.3 \mathrm{mg}, 90 \%)$ as white

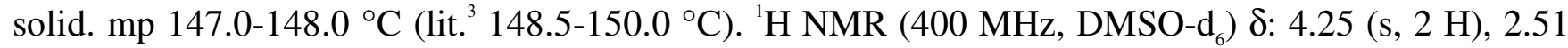
$(\mathrm{t}, J=7.4 \mathrm{~Hz}, 2 \mathrm{H}), 1.60-1.48(\mathrm{~m}, 2 \mathrm{H}), 1.32-1.23(\mathrm{~m}, 2 \mathrm{H}), 0.88(\mathrm{t}, J=7.3 \mathrm{~Hz}, 3 \mathrm{H})$.

\section{General experimental procedures for Suzuki cross-coupling reaction of 2,4-disubstituted-5-}

haloimidazole (Scheme 4): A degassed mixture of 2-(2',5'-Difluorophenyl)-4-phenyl-5-chloro-1Himidazole 19b (135.0 mg, $0.443 \mathrm{mmol}), \mathrm{Pd}_{2}(\mathrm{dba})_{3}(40.6 \mathrm{mg}, 0.0443 \mathrm{mmol})$, tri-tert-butylphosphonium tetrafluoroborate $(15.7 \mathrm{mg}, 0.0886 \mathrm{mmol})$, potassium phosphate $(282.1 \mathrm{mg}, 1.329 \mathrm{mmol})$, and organic boronic acid $(0.665 \mathrm{mmol})$ in toluene $(5 \mathrm{~mL})$ was heated at $100{ }^{\circ} \mathrm{C}$ for $16 \mathrm{~h}$. After being cooled to room temperature, the reaction mixture was diluted with EtOAc $(10 \mathrm{~mL})$, and then filtered. The filtration were concentrated. The residue was purified by flash chromatography (silica gel, hexane: EtOAc: TEA = 5: $1: 0.05)$ to give 2,4,5-trisubstituted-imidazole 23-25.

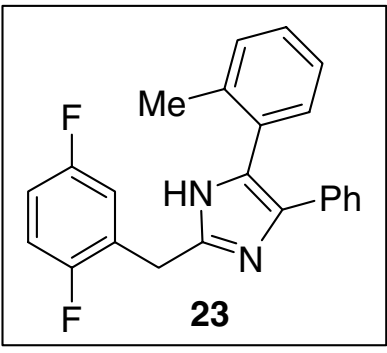
4-phenyl-1H-imidazole (23) 
Flash chromatography (silica gel, hexane: EtOAc: TEA = 6: 1: 0.05) afforded imidazole 23 (105.0 mg, $66 \%$ ) as white needles: mp 162.9-164.9 ${ }^{\circ} \mathrm{C}$. IR (thin film) $v_{\max } 3058,1653,1603,1559,1540,1497$, 1457, 1436, 1242, 1207, 907, 818, 763, 735, $695 \mathrm{~cm}^{-1} .{ }^{1} \mathrm{H}$ NMR (400 MHz, $\left.\left(\mathrm{CD}_{3}\right)_{2} \mathrm{CO}\right) \delta 11.00($ br s, 1 H), 7.40 (br d, $J=7.3 \mathrm{~Hz}, 2 \mathrm{H}), 7.30-7.25(\mathrm{~m}, 3 \mathrm{H}), 7.22-6.99(\mathrm{~m}, 7 \mathrm{H}), 4.05(\mathrm{~s}, 2 \mathrm{H}), 2.07(\mathrm{~s}, 3 \mathrm{H}) .{ }^{13} \mathrm{C}$ NMR (100 MHz, $\left.\left(\mathrm{CD}_{3}\right)_{2} \mathrm{CO}\right) \delta 158.6(\mathrm{dd}, J=240,2 \mathrm{~Hz}), 156.8(\mathrm{dd}, J=240,2 \mathrm{~Hz}), 144.2,141.5$, $137.4,130.7,130.2,128.6,128.2,128.0,127.3(\mathrm{dd}, J=20,10 \mathrm{~Hz}), 125.9,125.8,125.6,117.2(\mathrm{dd}, J=$ 20, $4 \mathrm{~Hz}), 116.3(\mathrm{dd}, J=30,10 \mathrm{~Hz}), 114.6(\mathrm{dd}, J=20,10 \mathrm{~Hz}), 27.2,19.2$. HRMS (ESI) calculated for $\mathrm{C}_{23} \mathrm{H}_{19} \mathrm{~F}_{2} \mathrm{~N}_{2}(\mathrm{M}+\mathrm{H})^{+} 361.1516$, found 361.1516.

\section{2-(2,5-Difluorophenyl)-5-[(1E)-hex-1-en-1-yl]-}

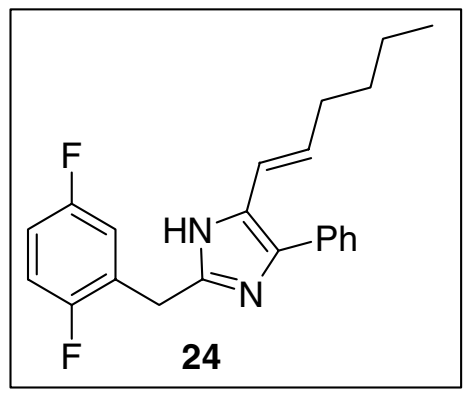

\section{4-phenyl-1H-imidazole (24)}

Flash chromatography (silica gel, hexane: EtOAc: TEA = 5: 1: 0.05) afforded imidazole 24 (93.0 mg, $60 \%$ ) as a yellow oil. IR (thin film) $v_{\max } 3017,1604,1530,1457,1433,1272,1242,1206,1178,960$, 868, 813, 768, $698 \mathrm{~cm}^{-1}$. ${ }^{1} \mathrm{H}$ NMR (400 MHz, $\left.\mathrm{CDCl}_{3}\right) \delta 9.00($ br s, $1 \mathrm{H}), 7.53(\mathrm{~d}, J=7.3 \mathrm{H}, 2 \mathrm{H}), 7.40$ $(\mathrm{t}, J=7.3 \mathrm{~Hz}, 2 \mathrm{H}), 7.30(\mathrm{t}, J=7.3 \mathrm{~Hz}, 1 \mathrm{H}), 7.10-7.00(\mathrm{~m}, 2 \mathrm{H}), 6.95-6.90(\mathrm{~m}, 1 \mathrm{H}), 6.45(\mathrm{~d}, J=15.9$ $\mathrm{Hz}, 1 \mathrm{H}), 6.25-6.00$ (m, $1 \mathrm{H}), 3.99$ (s, $2 \mathrm{H}), 2.25-2.13$ (m, $2 \mathrm{H}), 1.38-1.18$ (m, $4 \mathrm{H}), 0.89$ (t, $J=7.1 \mathrm{~Hz}$, $3 \mathrm{H}) .{ }^{13} \mathrm{C} \mathrm{NMR}\left(100 \mathrm{MHz}, \mathrm{CDCl}_{3}\right) \delta 158.7(\mathrm{dd}, J=240,2 \mathrm{~Hz}), 156.6(\mathrm{dd}, J=240,2 \mathrm{~Hz}), 144.6,133.9$, $128.9,128.5$ (2 C), 128.2, 127.3 (2 C), 126.9, 126.5, 126.2 (dd, $J=24,10 \mathrm{~Hz}), 117.3$ (dd, $J=20,4$ $\mathrm{Hz}), 116.2(\mathrm{dd}, J=24,10 \mathrm{~Hz}), 115.0(\mathrm{dd}, J=24,10 \mathrm{~Hz}), 32.8,31.5,28.3,22.2,13.9$. HRMS (ESI) calculated for $\mathrm{C}_{22} \mathrm{H}_{23} \mathrm{~F}_{2} \mathrm{~N}_{2}(\mathrm{M}+\mathrm{H})^{+} 353.1829$, found 353.1818 .

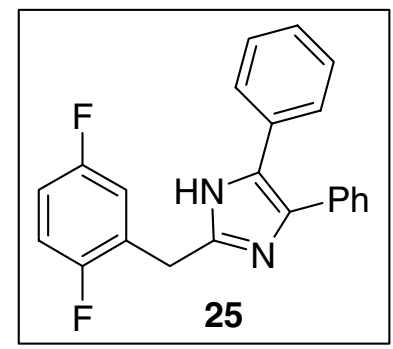


Flash chromatography (silica gel, hexane: EtOAc: TEA = 5: 1: 0.05) afforded imidazole 25 (105.0 mg, $71 \%$ ) as white needles: $\mathrm{mp} 206.3-206.5^{\circ} \mathrm{C}$. IR (thin film) $v_{\max } 3057,2931,2896,1559,1522,1437$, 1216, 820, 712, $699 \mathrm{~cm}^{-1} .{ }^{1} \mathrm{H}$ NMR (400 MHz, $\mathrm{CDCl}_{3}$ ) $\delta 9.92$ (br s, $\left.1 \mathrm{H}\right), 7.70-7.05$ (m, $10 \mathrm{H}$ ), 7.02$6.97(\mathrm{~m}, 1 \mathrm{H}), 6.94-6.87(\mathrm{~m}, 2 \mathrm{H}), 4.01(\mathrm{~s}, 2 \mathrm{H}) .{ }^{13} \mathrm{C} \mathrm{NMR}\left(100 \mathrm{MHz}, \mathrm{CDCl}_{3}\right) \delta 158.1(\mathrm{dd}, J=240,2$ Hz), 156.8 (dd, $J=240,2$ Hz), 144.7, 144.5 136.7, 135.8, 131.7, 128.5 (2 C), 127.9 (4 C), 127.4, 127.2 (2 C), 126.9 (dd, $J=44,13 \mathrm{~Hz}), 126.2,127.3(\mathrm{dd}, J=20,6 \mathrm{~Hz}), 126.3(\mathrm{dd}, J=25,10 \mathrm{~Hz})$, $114.6(\mathrm{dd}, J=25,10 \mathrm{~Hz}), 27.1$. HRMS (ESI) calculated for $\mathrm{C}_{22} \mathrm{H}_{17} \mathrm{~F}_{2} \mathrm{~N}_{2}(\mathrm{M}+\mathrm{H})^{+} 347.1360$, found 347.1359 .

\section{References}

1. Poupaert, J.; Bruylants, A.; Crooy, P. Synthesis, 1972, 622.

2. Sun, Y.; Martell, A. E.; Welch, M. J. Tetrahedron, 2000, 56, 5093.

3. Verschave, P.; Vekemans, J.; Hoornaert, G. Tetrahedron, 1984, 40, 2395.

4. Neugebauer, W.; Pine, E.; Kim, M.; Carey, P. R. Canadian J. Chem., 1996, 74, 341.

5. Lipshutz, B. H.; Hungate, R. W.; McCarthy, K. E. J. Am. Chem. Soc., 1983, 105, 7703.

6. Shiral, T.; Kurashige, S.; Nippon, K. Yuki Gosei Kagaku Kyokaishi, 1972, 30, 76.

7. Watanabe, T.; Ueda, I.; Hayakawa, N.; Kondo, Y.; Adachi, H.; Iwasaki, A.; Kawamata, S.; Mentori, F.; Ichikawa, M.; Yuasa, K.; Ohta, A.; Kurihara, T.; and Miyamae, H. J. Heterocyclic Chem. 1990, 27, 711 .

8. Griffiths, G. J., Hauck, M. B.; Imwinkelried, R.; Kohr, J.; Roten, C. A.; and Stucky, G. C. J. Org. Chem. Soc. 1999, 64, 8084.

9. Shi, Y.-J.; Frey, L. F.; Tschaen, D. M.; Verhoeven, T. R. Synth. Commun., 1993, 23, 2623. 